\title{
LXRs are finally being adequately targeted in atherosclerosis
}

\author{
Julio Buñay $^{1,2}$, Silvère Baron ${ }^{1,2}$, Jean-Marc A. Lobaccaro ${ }^{1,2}$ \\ ${ }^{1}$ Université Clermont Auvergne, GReD, CNRS UMR 6293, INSERM U1103, 28, place Henri Dunant, BP38, F63001, Clermont-Ferrand, France; \\ ${ }^{2}$ Centre de Recherche en Nutrition Humaine d'Auvergne, 58 Boulevard Montalembert, F-63009 Clermont-Ferrand, France \\ Correspondence to: Jean-Marc A. Lobaccaro; Silvère Baron. Université Clermont Auvergne, GReD, Faculté de Médecine, 28 place Henri-Dunant, \\ BP38, F-63001 Clermont-Ferrand, France.Email: j-marc.lobaccaro@uca.fr; silvere.baron@uca.fr \\ Comment on: Guo Y, Yuan W, Yu B, et al. Synthetic High-Density Lipoprotein-Mediated Targeted Delivery of Liver X Receptors Agonist Promotes \\ Atherosclerosis Regression. EBioMedicine 2018;28:225-33.
}

Submitted Aug 03, 2018. Accepted for publication Sep 12, 2018.

doi: $10.21037 / \mathrm{atm} .2018 .09 .35$

View this article at: http://dx.doi.org/10.21037/atm.2018.09.35

Atherosclerosis is the major cause of morbidity and mortality (1). Schematically, plaque is mainly made of fat, cholesterol and calcium, builds up in the arteries, and limits the oxygen-rich blood flow. Serious problems obviously occur when the arteries become narrow and/or become too closed, enough to impede blood flow. The exact cause of atherosclerosis is unknown; however, numerous risk factors have been identified such as diabetes, obesity, high blood pressure, cigarette usage and an unbalanced diet, especially those enriched in lipids. This is because cholesterol increases within the arterial walls has been clearly associated with a higher risk of developing atherosclerosis. It has become evident that decreasing such accumulations could be associated to a lower risk of developing this disease (1). Indeed, numerous meta-analysis studies pointed out the beneficial effects of lowering cholesterol which was associated with LDL, for example with statins, HMGCoA reductase inhibitors which blocks de novo synthesis of cholesterol. Cholesterol homeostasis is schematically balanced between two main "compartments", the liver and the non-hepatic peripheral tissues. Low density lipoprotein (LDL) carries the cholesterol from the liver to the nonhepatic tissue, while cholesterol is transported from nonhepatic tissue by high-density lipoproteins (HDL) through the so-called reverse cholesterol transport (RCT). Early formations of atherosclerotic plaque, involves a LDLcholesterol increase, which accumulates in the endothelium and become oxidized. This oxidized-LDL is then absorbed by differentiated macrophages and slowly transforming into "foam"-cells (2). Because foam cells are not able to regulate cholesterol accumulation, they will eventually die. This further propagates the inflammatory process by secreting cytokines which recruit circulating monocytes, that will differentiate into macrophage. A vicious circle thus emerges (Figure 1A).

Interestingly, many transcription factors are sensitive to the intracellular levels of cholesterol. Among these, the liver $\mathrm{X}$ receptors (LXR $\alpha / \mathrm{NR} 1 \mathrm{H} 3$ and $\mathrm{LXR} \beta / \mathrm{NR} 1 \mathrm{H} 2)$ have a prominent place. These nuclear receptors, which were identified in the mid 90's, were initially classified as cholesterol-lowering factors through the phenotypic analysis of various strains of engineered mice deficient for one or both LXRs. For a full review see (3). Interestingly LXRs are activated by endogenous ligands, derived from enzymatic oxidation of cholesterol, named oxysterols. Hence, when cholesterol concentration increases within the cell, oxysterols bind to LXR $\alpha$ or LXR $\beta$, which heterodimerizes with one of the three RXRs/NR2B1-3, receptors for the 9-cis retinoic acid, on specific sequences located within the promoters of target genes. Following the binding of their bona fide ligands, LXR/RXR recruits protein co-activators and the transcriptional machinery, which will in turn increase the transcription rate of the target genes. The reason LXRs have been defined as hypocholesterolemiant proteins, lies in their targets. Indeed, LXRs increase the efflux of cholesterol by the ATP-binding cassette $\mathrm{A} 1$ (ABCA1) transporter. ABCA1 is a membrane protein that transfers cholesterol to Apolipoprotein A1 (ApoA1), the major component of HDL present in the macrophages. The gene encoding the cholesteryl ester transfer protein (CETP), which favors the transfer of esterified cholesterol to the other lipoproteins such as very 

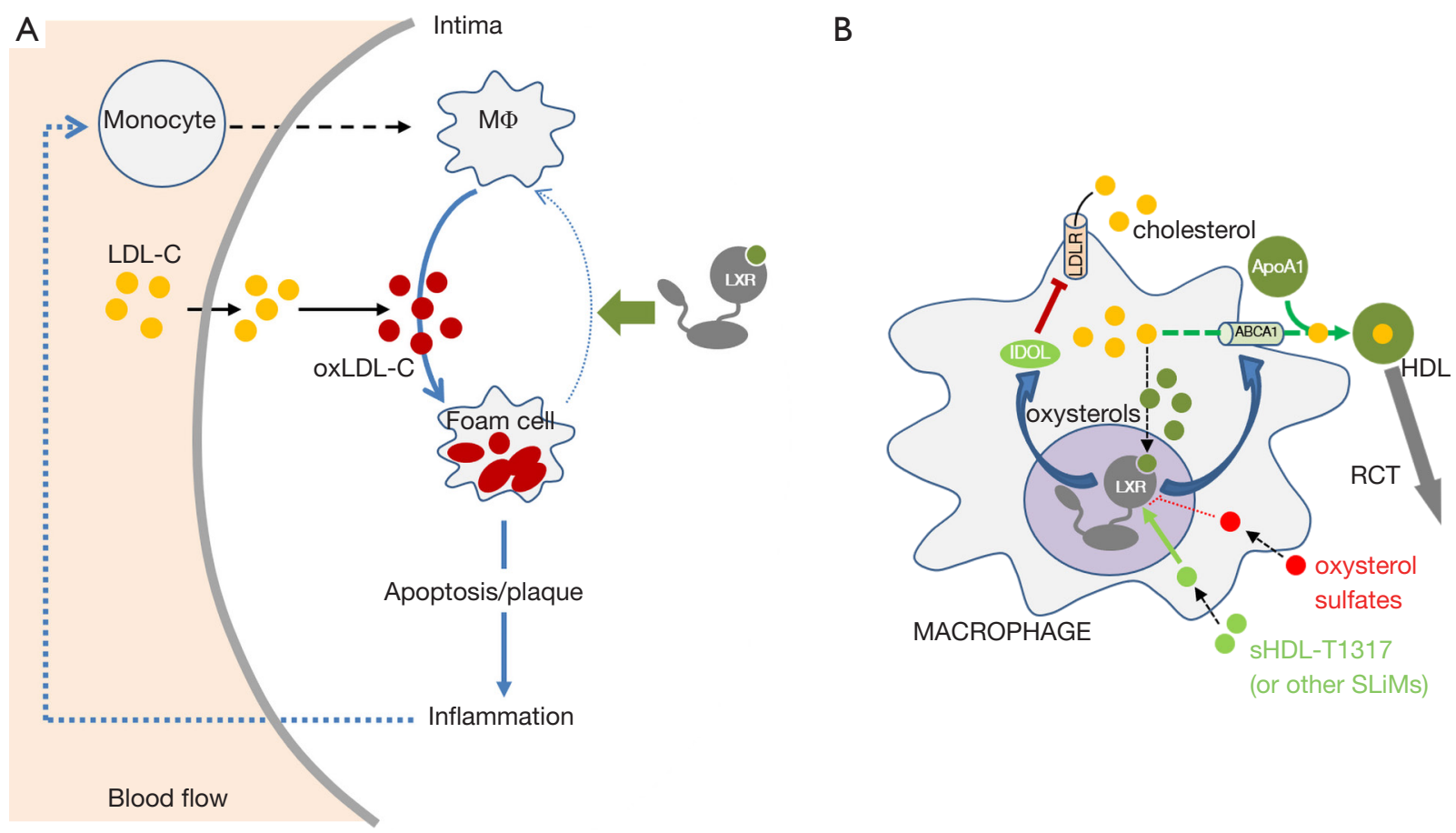

Figure 1 Development of the atherosclerotic plaque and effect of LXR activation in the macrophage on the cholesterol homeostasis. (A) When LDL (LDL-C) increases in the blood flow, its concentration is higher in the intima of the arterial walls and its oxidation is favored. This oxidized LDL (oxLDL-C) is absorbed by the macrophages (MФ), which are transformed into foam cells, full of cholesterol. As these cells are unable to control their cholesterol homeostasis, they enter into apoptosis and secrete inflammatory factors, increasing the recruitment of circulating monocytes in the intima. (B) Activating the nuclear receptors LXRs should promote the efflux of the cholesterol by the ABCs proteins before the macrophages transform into foam cells. Due to, when cholesterol increases, it is transformed into oxysterols. The binding to LXRs with its endogenous ligand or with synthetic LXR agonist complexed with sHDL, such as T0901317 or another selective liver X modulators (SLiMs), will induce two main responses for controlling cholesterol levels: (I) IDOL, a ubiquitin ligase targeting the LDL-receptor (LDLR) is enhanced, which will decrease LDL-cholesterol influx; (II) ABCA1 (as well as ABCG1 non-indicated in this Figure) accumulation is induced as well as cholesterol efflux. Cholesterol will be taken by ApoA1 to form the HDL and in this way favors the reverse cholesterol transport (RCT) to the liver and the atherosclerosis regression. For more details, please refer to the main text.

low-density lipoproteins (VLDL), is also a target of LXRs. At last, LXRs also decrease the cholesterol influx by the LDL-receptor (LDLR) by the ubiquitin ligase IDOL that targets LDLR. As this protective mechanism physiologically exists in the cells, macrophages should never be able to transform into foam cells and the cholesterol homeostasis would always be controlled (Figure 1B). It was hypothesized that a sulfate-derived oxysterols, predominantly found in the atherosclerotic plaque, act as a LXR inhibitor, and thus impedes the increase of the RCT by the macrophages (4). Once again, a vicious circle is created as the cholesterol accumulation within the macrophages cannot be ruled out. The dead foam cells that accumulate into the plaque, will favor the sulfatation of oxidized cholesterol, and finally block the positive effects of LXRs in macrophages, decreasing the cholesterol accumulation (Figure 1B). Furthermore, the activation of LXRs would also influence the improvement of arteriosclerosis by the suppression of an inflammatory signaling on the macrophages (5).

Based on that, it is understandable that the synthetic LXR agonists have been the center of active research from pharmaceutical companies, not only to fight atherosclerosis but also many various other metabolic or neurological diseases, as well as cancers (6). However, the synthetic ligands that have been developed so far, have been associated with important side effects, such as hypertriglyceridemia and neurological disorders. These side effects have been associated to the absence of a specific delivery method of the 
drugs. We and others have postulated that the development of selective ligands, we called SLiMs for selective Liver X receptor modulators (7), would probably be the future of the LXR pharmacology that is usable for human therapy. These SLiMs should be specific for each LXR isoform, able to recruit co-activators that are involved in a targeted metabolic pathway, e.g., the cholesterol homeostasis, without modifying other pathways, e.g., fatty acid synthesis or glucose homeostasis. The first synthetic compound with some of these characteristics was reported by Makishima's lab (8). YT-32 was derived from ergosterol, and was able to decrease the level of circulating cholesterol in mice, without inducing any hypertriglyceridemia.

The paper published by Guo et al. (9) used a different approach to deliver the LXR agonist, T0901317. This molecule was the historically first synthetic LXR agonist (10); however, it also had numerous side effects such as an increase of the fatty acid synthesis by the liver (6). These deleterious and unexpected observations blocked its use in human therapy. Indeed, T0901317 is able to target the macrophages, increase ABAC1 and the RCT; on the other side when uptaken by the liver, this compound also activates LXRs and their downstream target genes, acylCo-A carboxylase (ACC), fatty acid synthase (FAS), sterol response element binding protein (SREBP) 1c and stearoyl Coenzyme A desaturase 1 (SCD1), all of them of which are involved in triglyceride homeostasis. To specifically target macrophages, the T0901317 was encapsuled in synthetic HDL nanoparticles (sHDL). The authors tested the effects of this new delivery system in vitro, and observed that the established macrophage cell line J774A.1 was able to promote the cholesterol efflux by increasing $A b c a 1$ and $A b c g 1$ expression. Interestingly, the cholesterol efflux was directed to the HDL particles. Not only in vitro, these synthetic HDL nanoparticles were able to target the atherosclerotic plaque, and more importantly, to increase $A b c a 1$ and $A b c g 1$ expression in the aorta, demonstrating the usefulness of this molecular tool to specifically deliver the LXR agonist. Finally, the most impressive result came from the treatment of a mouse model with atherosclerosis which looked at ApoE-deficient mice that were fed a high cholesterol diet to develop atheroma. Once the plaque was present, some mice were treated with the sHDLT1317. Compared to the vehicle-treated mice, the nanoparticlederived T0901317 agonist treated mice showed a decrease of aortic plaque, an activation of $A b c a 1$ and $A b c g 1$, and no modification of the hepatic lipogenesis, even after a longterm treatment.
This promising new approach to deliver specific drugs to the macrophages within the plaque opens up incredible new opportunities. First, this demonstrates that it is possible to specifically target LXRs in order to favor the RCT with the lowest side effects, which up to now have impeded the use of synthetic LXR agonists to treat atherosclerosis. Second, this method will also be useful to deliver even more potent and specific LXR agonists and/or other drugs in association. Indeed, the results obtained in vivo are important in terms of hope for the development of new drugs and new celltargeted therapies. However still modest; the effect of newly developed LXR agonists will have to be investigated.

\section{Acknowledgements}

The authors would like to thank the lab members for their critical reading of the mauscript.

Funding: Part of this work was supported by grants from Région Auvergne Rhône Alpes, Fond Européen de Développement Régional (FEDER), AAP Plan Cancer Environnement 2016, PNRP (13-MRES-PNRPE-1CVS043).

\section{Footnote}

Conflicts of Interest: The authors have no conflicts of interest to declare.

\section{References}

1. WHO/2011 Global atlas on cardiovascular disease prevention and control [Internet]. Available online: http:// www.who.int/cardiovascular_diseases/publications/atlas_ cvd/en/

2. Shibata N, Glass CK. Macrophages, oxysterols and atherosclerosis. Circ J 2010;74:2045-51.

3. Maqdasy S, Trousson A, Tauveron I, et al. Once and for all, LXR $\alpha$ and LXR $\beta$ are gatekeepers of the endocrine system. Mol Aspects Med. 2016;49:31-46.

4. Cook IT, Duniec-Dmuchowski Z, Kocarek TA, et al. 24-hydroxycholesterol sulfation by human cytosolic sulfotransferases: formation of monosulfates and disulfates, molecular modeling, sulfatase sensitivity, and inhibition of liver $\mathrm{x}$ receptor activation. Drug Metab Dispos Biol Fate Chem 2009;37:2069-37:2

5. Lee SD, Tontonoz P. Liver X receptors at the intersection of lipid metabolism and atherogenesis. Atherosclerosis 2015;242:29-36. 
6. Viennois E, Pommier AJC, Mouzat K, et al. Targeting liver $\mathrm{X}$ receptors in human health: deadlock or promising trail? Expert Opin Ther Targets 2011;15:219-32.

7. Viennois E, Mouzat K, Dufour J, et al. Selective liver X receptor modulators (SLiMs): what use in human health? Mol Cell Endocrinol 2012;351:129-41.

8. Kaneko E, Matsuda M, Yamada Y, et al. Induction of intestinal ATP-binding cassette transporters by a phytosterol-derived liver X receptor agonist. J Biol Chem 2003;278:36091-278

9. Guo Y, Yuan W, Yu B, et al. Synthetic High-Density

Cite this article as: Buñay J, Baron S, Lobaccaro JA. LXRs are finally being adequately targeted in atherosclerosis. Ann Transl Med 2018;6(Suppl 1):S28. doi: 10.21037/atm.2018.09.35
Lipoprotein-Mediated Targeted Delivery of Liver X Receptors Agonist Promotes Atherosclerosis Regression. EBioMedicine 2018;28:225-33.

10. Repa JJ, Liang G, Ou J, et al. Regulation of mouse sterol regulatory element-binding protein-1c gene (SREBP-1c) by oxysterol receptors, LXRalpha and LXRbeta. Genes Dev 2000;14:2819-14.

(English Language Editor: Jeremy Dean Chapnick, AME Publishing Company) 